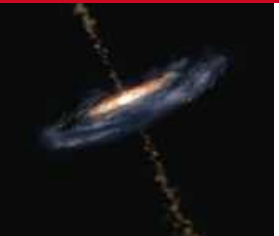

HIGH-ENERGY COSMIC RAYS TRACED TO SOURCE Supermassive blackhole galaxies spin out super-energetic particles. www.nature.com/news

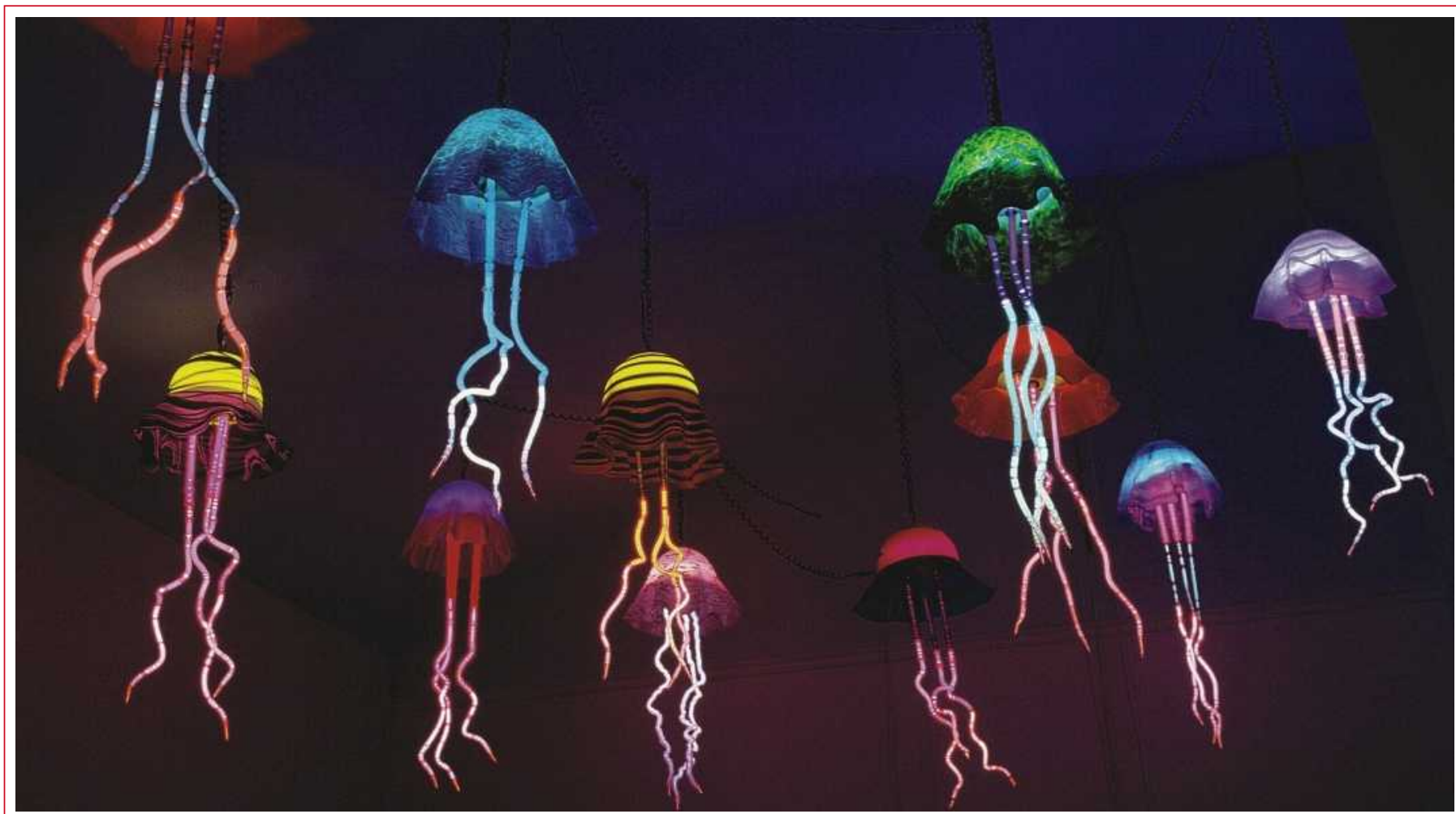

\section{SNAPSHOT}

\section{Light relief}

Luminous jellyfish sculptures fill an exhibition room at the Katonah Museum of Art in New York. The metre-long works by New Orleans artist Eric Ehlenberger consist of blown-glass bodies attached to three neon-filled tentacles powered by a 12 -volt d.c. adaptor plugged into a standard electric outlet. Each jellyfish sells for US $\$ 2,250$. emergency-room physician, creates abstract and figurative works that focus on marine life, including fish, dolphins and whales. He was recently commissioned by the Seattle Biomedical Research Institute - which is working on a malaria vaccine - to design a neon sculpture illustrating the feeding posture of anopheline mosquitoes.

The exhibition, called The

Shattering Glass: New Perspectives, runs until 24 February 2008.

Nick Thomas

\title{
Brain waves reveal intensity of pain
}

\section{SAN DIEGO}

Recordings from electrodes in the human brain may offer the first objective way to measure the intensity of pain. Researchers say that they have found a neural signal that correlates with the amount of pain that an individual feels. The signal could be used to refine painrelief techniques that involve stimulating the brain with electricity, they say.

Single cells have previously been identified in the human brain that are active in pain, but their response is binary, signalling either pain or no pain. Now, Morten Kringelbach of the psychiatry department at the University of Oxford, UK, and his colleagues have identified low-frequency brain waves that emanate from two regions buried deep within the brain when a patient is in pain. The more pain that is experienced, the longer the waves last.

Kringelbach's team recorded activity from two electrodes positioned in the thalamus and the periaqueductal grey area of 12 awake people who had been undergoing deep-brain stimulation (DBS) for chronic pain. During the recording, the team touched either a painful or pain-free area of the patients' bodies and had patients rate their pain every minute. The duration of the waves - dubbed "pain spindles" — correlated with how intensely the patients felt their pain. "It is an objective measure that correlates with a subjective measure," says Kringelbach, who presented the findings at the Society for Neuroscience meeting in San Diego, California, last week.

This signal could help refine DBS for such patients, he says. Currently, the electrodes fire signals continuously, even when the patient doesn't need pain relief, which wears down the implanted battery. "We could have a stimulator that picks up this neural signature of pain and only starts sending signals at that point," Kringelbach says.

"It would be great to have a 'signature of

\section{"It is an objective} measure that correlates with a subjective measure." pain'," says Allan Basbaum, a neuroscientist at the University of California, San Francisco. It is not clear from this preliminary study whether these spindles disappear when painkillers, anaesthetics or electrical stimulation are used, he adds.

The next step is to try to record the signals with a non-invasive technique, such as magnetoencephalography, which measures electrical activity in the brain via changes in the magnetic field. This could allow pain monitoring in a much broader range of patients.

Kerri Smith 